\title{
Opalescence: Tooth Whitening Systems
}

Dan Fischer, Tanye, Chantell Thomson,T. Schreuder

Dan Fischer, Tanye, Chantell Thomson, T. Schreuder: Opalescence: Tooth Whitening Systems. Tanz Dent J. 2009; 15 (2):48-53

Correspondence: Chantell Thomson, E-mail: Chantell@wright-millners.co.za

\section{Ultradent}

Ultradent celebrates 30 years of success. It was developed by Dr Dan Fisher who is a dentist. Its Head office is based in America, Utah. The Opalescence tooth whitening range has been around for 21 years. It is approved by American Dental Association (ADA). Ultradent Tooth whitening products were re-launched in May 2008.

\section{History of Whitening}

The Opalescence tooth whitening was discovered accidentally by an Orthodontist, Dr.Bill Klushmeyer, in the late 60's. The chemical, Glyoxide, used for antiseptic purpose, also bleached the teeth.

\section{Why tooth whitening}

Tooth whitening is done for either the patient or practitioners' satisfaction requirements. For the patient, appearance of being more healthy, youthfulness and over all good feeling are the main attributes. Whereas for the dental practitioner job satisfaction, extra income and advertisement are the reasons behind performing tooth whitening.

There are several causes of tooth discoloration that may lead to a need of tooth whitening. These may be external or internal.

\section{External causes include:}

- Drinks (colas, coffee, tea, wine)

- Food colorants (beetroot, curries)

- Tobacco (cigarettes, chewing)

- $\quad$ Aging (thinning of enamel)

\section{Internal causes are:}

- Drugs (tetracycline, fluoride)

- Trauma (Non vital tooth)

- $\quad$ Systemic conditions

\section{Limitations}

Young child (four years and younger) as well as pregnant or lactating women.

\section{How safe is whitening?}

Hundreds of studies from the most creditable researchers have proved that there is: no enamel reduction, no enamel softening and no damage or change in tooth structure.

\section{Sensitivity from Whitening}

This may present as dull toothache or headache which is caused by tooth dehydration. The symptom may be solved by breaking from treatment for a day or two, shorten bleaching time, or use a desensitizing gel containing fluoride, or potassium nitrate and fluoride.

\section{How does the Potassium Nitrate and Fluoride (PF) stop sensitivity?}

Potassium nitrate acts more like an analgesic or anesthetic by preventing the nerve from repolarizing after it has depolarized in the pain cycle while the fluoride occludes the tubules.

\section{$38 \%$ hydrogen peroxide}

- Maximum strength whitens faster than any other formula

\section{No light required}

- Lights produce dehydration from heat. This makes the teeth look overly white at first, but the teeth darken after a few days and disappoint the patient.

- Lights can cause heat that does damage to tissues or causes discomfort

- Bleaching lights are expensive

- Bleaching lights take up space in the operatory 


\section{How does it work?}

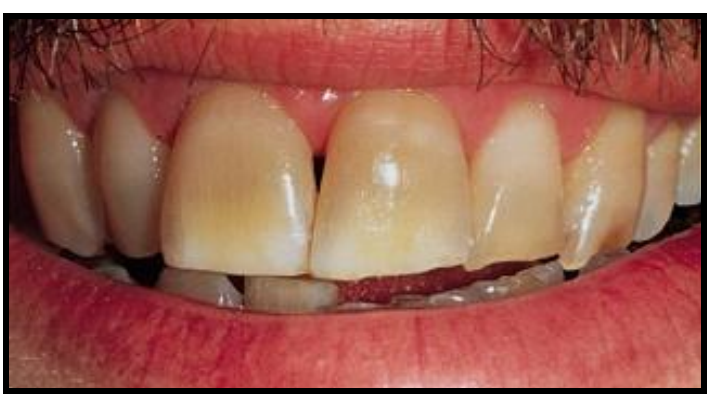

Before

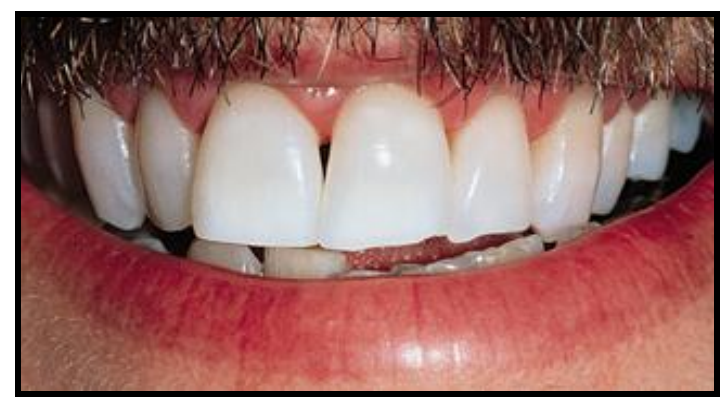

After

(Treatment may take 2-6 months.)

Tooth whitening is more than dental cosmetics.

\section{Chemically activated}

Freshly mixed product

- $\quad$ No expensive light required

\section{Gel consistency}

Won't drip after placement

\section{Syringe-to-syringe mixing}

- $\quad$ Rapid and mess free

- $\quad$ Prevents assistants from burning their fingers

\section{Bright red color}

Easy to see during placement and removal; doctors are aware if it has been accidentally expressed onto tissue

Apply a $0.5 \mathrm{~mm}$ layer of Opalescence Xtra Boost

- $\quad$ Allow material to rest on the teeth for a total of 10-15 minutes

If patient experiences sensitivity, remove material sooner

The procedure can help change people's lives.
- $\quad$ For maximum effectiveness, stir gel on teeth every 5 minutes

Suction gel using a Luer Vacuum Adapter and a SurgiTip. This controlled suction prevents dislodgement of OpalDam.

Reapply Opalescence Xtra Boost if additional whitening is desired - up to 3 applications in one visit.

One syringe of material should be sufficient for 2-3 patients, with 3 applications on each arch.

Lift OpalDam from surface using an explorer or instrument.

Remove interproximally using explorer and/or floss.

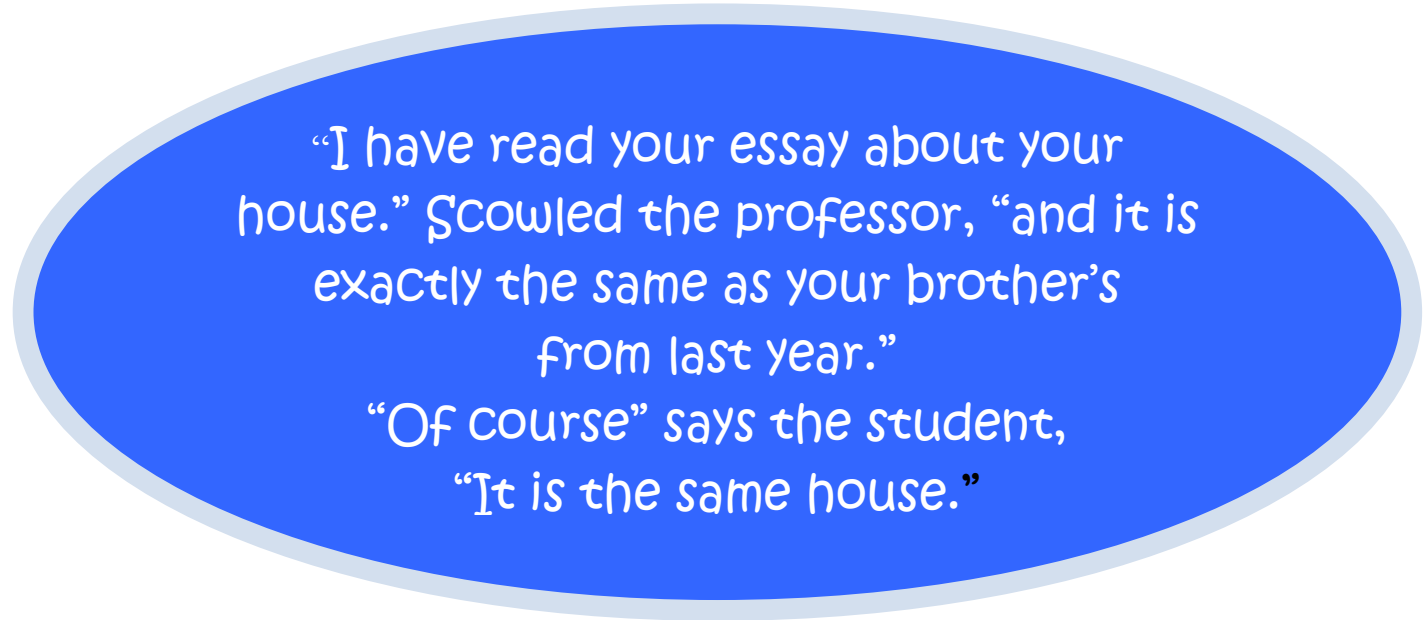




\section{Opalescence Trèswhite Supreme}

Disposable whitening trays

-10\% hydrogen peroxide

- Potassium nitrate and fluoride

-Disposable

- One size fits all

-Preloaded whitening trays

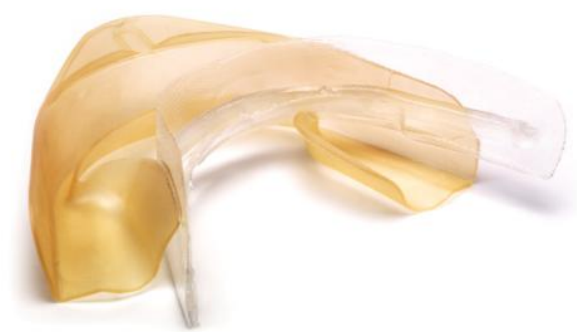

Enhanced tray design a sleeker more comfortable fit

Disposable, one-size-fits-all, preloaded whitening trays

Faster, superior results

Delicious improved flavors: mint melon and peach

Place tray in mouth

Suck down slightly

Remove outer tray

Leave in for $30-60 \mathrm{~min}$

Some doctors let the patients wear the tray for up to 2 hours - but not more

Research shows that once bleaching molecules enter the tooth, they go in all directions and penetrate the entire tooth. You couldn't localize bleaching to an isolated area even if you wanted to. There is plenty of research - that will back this up.

Bleaching will not disturb existing bonds (brackets or composite restorations). Bleaching will only

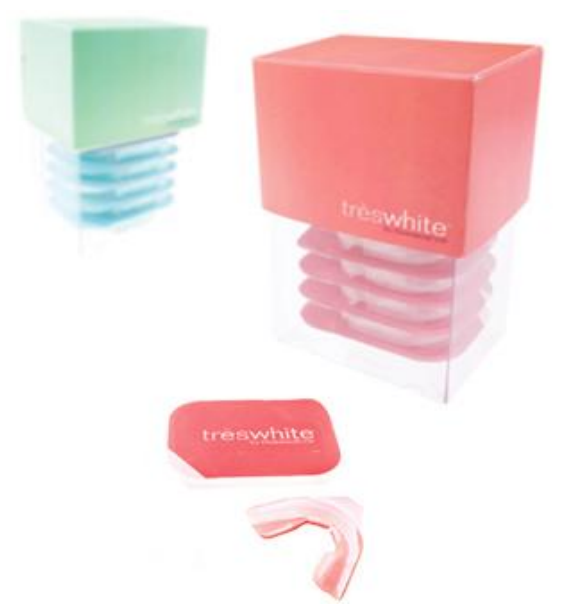

inhibit the adhesion of new materials performed in procedures within two weeks following the bleaching treatment.

\section{Three concentrations}

- Allows clinician to customize a treatment for specific patient needs

\section{Sticky gel consistency}

- Gel holds tray in place

- $\quad$ Less wasted gel

- $\quad$ Limited material swallowed by patient

\section{$\mathbf{2 0 \%}$ water content}

- $\quad$ Minimizes dehydration that can lead to sensitivity

\section{Three excellent flavors}

- Encourages patient compliance Increases satisfaction

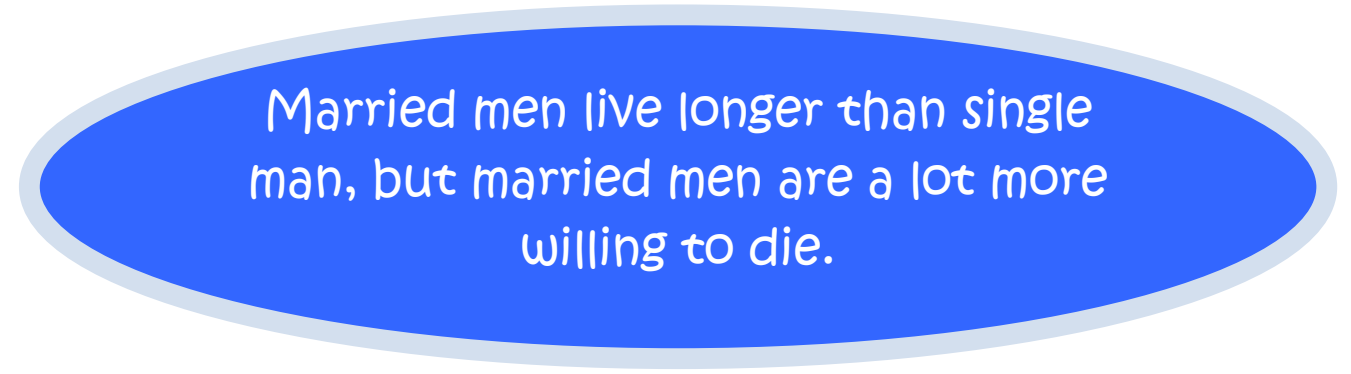




\section{Opalescence \\ Carbamide peroxide tooth whitening gels}

- Four concentrations - 10\%, 15\%, 20\%, 35\%

- Available with desensitizing agents $-0.5 \%$ potassium nitrate, $0.11 \%$ ion fluoride

- Patented, sustained-release formula

- Sticky gel consistency

- Soft tray with reservoirs

- $20 \%$ water content

- Three excellent flavors

Opalescence is active for $8-12$ hours

- After 2 hours the peroxide is till $40 \%$ active

- Tooth whitening is more about contact time than peroxide $\%$

\section{Opalescence Quick}

Waiting room whitener $45 \% \mathrm{CP}$

- Patient wears 30 min to 1 hour in custom tray

- “Jump Start" Whitening

- Good for first evaluation of patient's reaction

- Follow up with take home

- $\quad$ Touch Up Whitening

- As maintenance procedure

$45 \%$ carbamide peroxide

- $\quad$ Stick gel consistency

- $\quad$ Patented sustained release formula

- $10 \%$ water content

- $\quad$ Soft, thin tray with reservoirs

- $\quad$ Store in refrigerator

\section{In-Office Power Whitening}

- Time commitment - on the dentist's side

- Higher cost due to chair time

- Shorter treatment, stronger concentration

How does bleach work under ortho brackets?

Because the hydrogen peroxide easily penetrates the tooth and moves in different directions, even areas of the tooth that are not covered in bleach will whiten.

\section{How does bleaching affect immediate bond strengths?}

- It compromises the bond strength but only for 7-10 days

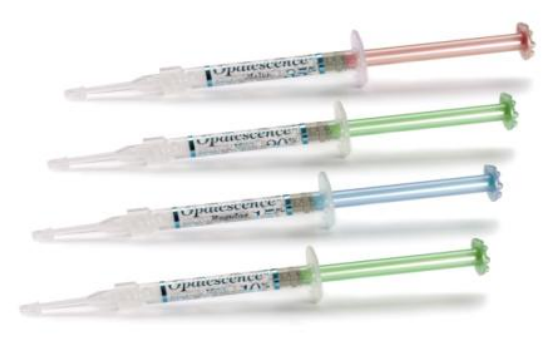

Opalescence Boost with brackets

- These brackets were debonded two weeks following the whitening treatment. Research shows that once bleaching molecules enter the tooth, they go in all directions and penetrate the entire tooth. You couldn't localize bleaching to an isolated area even if you wanted to. There is plenty of research - that will back this up.

- Bleaching will not disturb existing bonds (brackets or composite restorations). Bleaching will only inhibit the adhesion of new materials performed in procedures within two weeks following the bleaching treatment.

\section{Tray Fabrication}

Flame polish edges of tray and adapt with wet finger

- Smooths rough edges that could irritate gingiva

A quality impression is the first step to a customfabricated tray.

Chromaclone is a fast-setting, color-changing alginate.

\section{Color-changing formula}

- Indicates proper mixing, loading, and tray insertion time

- $\quad$ Reduces waste

- Takes away guessing

Formulated with a stiff consistency

- $\quad$ Reduces patient gagging

\section{Quick set time}

- Saves time 
- $\quad$ Reduces patient gagging

- Pour up stone model

- Cut model in a horseshoe shape, removing palatal and tongue areas

- Use LC Block-Out to create reservoirs on model (0.5mm layer)

- Do not apply block out material to incisal edge, interproximal areas, or next to the gingival margin. Doing so will allow bleaching material to escape from the bleaching tray.

- Other manufacturers do not recommend reservoirs. Opalescence will be effective with or without reservoirs, but like other bleaching materials, it will work more quickly when reservoirs are used.

- Light cure block out material in a curing oven or with a light cure unit

Teeth marred by discoloration or brown markings due to fluorosis may also be bleached. The mild cases can result in tiny white specks or streaks.

\section{Opalustre}

Enamel Microabrasion Slurry

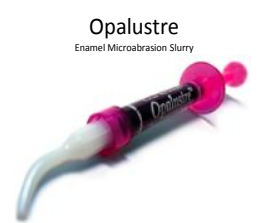

Contains $6.6 \%$ hydrochloric acid and silicon carbide microparticles

- Combination of acid and abrasive ingredients allow for chemical and mechanical stain removal

- Allows for one-appointment results

\section{Optimum viscosity}

- $\quad$ Ease of application to specific areas.

\section{Convenient syringe delivery}

- Syringe applicator provides control, ease of use, and direct placement into difficult-toaccess areas.

\section{Distinct purple color}

- Unique, dark purple color assists in accurate placement of material

\section{May be followed with OpalCups finishing cups}

- Provides natural enamel glazed surface

\section{Opalustre Enamel microabrasion slurry}

- $6.6 \%$ hydrochloric acid and silicon carbide microparticles
- Prophy cups with hard bristles to reduce splatter

- Different staff members may be able to perform procedure

- Optimum viscosity

- Convenient syringe delivery

- Distinct purple color

- ADA insurance code

- $\quad$ 9970, enamel microabrasion

\section{Opalustre Enamel microabrasion slurry}

Apply a fine-grit, water-cooled diamond bur to the stained enamel region for 5 to 10 seconds to initiate microreduction. For difficult staining, scratch the surface with a diamond bur, opens the stain to receive the product

Apply a $1.0 \mathrm{~mm}$ layer of Opalustre over labial surfaces.

Opalescence Endo Walking bleach for non-vital teeth

$35 \%$ hydrogen peroxide

- High concentration allows for a faster whitening procedure

Paste-like consistency

- $\quad$ Easily placed inside pulp chamber

Active in pulp chamber 3-5 days

- Infrequent change of material; less chair time

White color (almost clear)

- $\quad$ Easily visible white color inside tooth

- Won't show through tooth/alter appearance

- Won't stain inside of tooth

Pre-mixed, syringe delivered

Saves time

- $\quad$ Less wasted material

- Reduces chance of spillage or injury to patient or clinician from mixing

Easily dispensed

Opalescence Endo Walking bleach for non-vital teeth

Create access in lingual aspect of tooth

Remove restorative material to $2.0 \mathrm{~mm}$ apical to cemento-enamel junction

- Mix a small amount of glass ionomer to a putty and form a $1.0 \mathrm{~mm}$ sealing plug

Express Opalescence Endo into chamber, leaving 1.0 to $1.5 \mathrm{~mm}$ of space for the provisional

Place a non-eugenol provisional restorative material (like UltraTemp) to seal the access opening

Wipe off excess with a wet cotton swab 
- $\quad$ Leave Opalescence Endo in the chamber for 35 days and repeat if necessary

\section{UltraEZ}

Sustained-release desensitizing gel

- $3 \%$ potassium nitrate

- $0.11 \% \mathrm{w} / \mathrm{w}$ fluoride ion

- Sticky gel consistency

- Sustained release formula

- Unit dose syringes

Contains $3 \%$ potassium nitrate and $0.11 \%$ w/w fluoride ion

- Affords quick and long-lasting relief of sensitivity

Sticky gel consistency

- Gel holds tray in place

- Less wasted gel

- $\quad$ Limits material swallowed by patient

\section{Sustained release}

- Effective for duration of treatment

\section{Unit dose syringes}

- Easy for patient to dispense

\section{Some uses of UltraEZ}

- Pre/post bleaching sensitivity

- Sensitivity from toothbrush abrasion

- Periodontal treatments i.e. root planing

- General hot/cold sensitivity

- $\quad$ Sensitivity from prophy cleaning

\section{Opalescence Toothpaste}

Though Opalescence Toothpaste contains the same amount of fluoride as leading brands, its formula allows the tooth to absorb over four times more fluoride.

Set up your shade guide Shade Guide by Hue

A1-A2-A3-A3.5-B1-B2-B3-B4-C1-C2-C3-C4-D1D2-D3-D4...

Not good for whitening

Shade Guide by Value

B1-A1-B2-D2-A2-C1-C2-D4-A3-D3-B3-A3.5-B4-

$\mathrm{C} 3-\mathrm{A} 4-\mathrm{C} 4$

First is classified by values

Second is classified by hue

\section{A young lady boarded a crowded bus and requested a gentleman to vacate a seat saying she is pregnant. The man soon gave his seat and looked at her saying she does not look pregnant. "Yes, she replied with a smile. "It has only been about an hour!"}

Teacher: Why are you late?

Wilson: Because of the sign.

Teacher: What sign?

Wilson: The one that says "School ahead go slow" that is what I did. 